\title{
A THREE-DIMENSIONAL FINITE ELEMENT MODEL OF AN ADHERENT EUKARYOTIC CELL
}

\author{
J. G. McGarry and P.J. Prendergast*
}

Centre for Bioengineering, Department of Mechanical Engineering, Trinity College Dublin, Ireland

\begin{abstract}
Mechanical stimulation is known to cause alterations in the behaviour of cells adhering to a substrate. The mechanisms by which forces are transduced into biological responses within the cell remain largely unknown. Since cellular deformation is likely involved, further understanding of the biomechanical origins of alterations in cellular response can be aided by the use of computational models in describing cellular structural behaviour and in determining cellular deformation due to imposed loads of various magnitudes. In this paper, a finite element modelling approach that can describe the biomechanical behaviour of adherent eukaryotic cells is presented. It fuses two previous modelling approaches by incorporating, in an idealised geometry, all cellular components considered structurally significant, i.e. prestressed cytoskeleton, cytoplasm, nucleus and membrane components. The aim is to determine if we can use this model to describe the non-linear structural behaviour of an adherent cell and to determine the contribution of the various cellular components to cellular stability. Results obtained by applying forces (in the picoNewton range) to the model membrane nodes suggest a key role for the cytoskeleton in determining cellular stiffness. The model captures non-linear structural behaviours such as strain hardening and prestress effects (in the region of receptor sites), and variable compliance along the cell surface. The role of the cytoskeleton in stiffening a cell during the process of cell spreading is investigated by applying forces to five increasingly spread cell geometries. Parameter studies reveal that material properties of the cytoplasm (elasticity and compressibility) also have a large influence on cellular stiffness. The computational model of a single cell developed here is proposed as one that is sufficiently complex to capture the non-linear behaviours of the cell response to forces whilst not being so complex that the parameters cannot be specified. The model could be very useful in computing cellular structural behaviour in response to various in vitro mechanical stimuli (e.g. fluid flow, substrate strain), or for use in algorithms that attempt to simulate mechanobiological processes.
\end{abstract}

Key words: Cell model, finite elements, tensegrity, microtubules, actin, prestress, cellular deformation, nuclear deformation, mechanobiology, cell biomechanics.

*Address for correspondence:

Patrick Prendergast

Centre for Bioengineering,

Department of Mechanical Engineering,

Parsons Building, Trinity College, Dublin 2, Ireland.

Telephone Number: +353-1-608 1383

E-mail: pprender@tcd.ie

Web.: www.biomechanics.ie
Introduction

Alterations in cell shape and structure caused by mechanical loads are critical to cell functions including growth, motility, differentiation, and proliferation (Chen et al., 1997; Chicurel et al., 1998; Janmey, 1998; Carter and Beaupré, 2003). Much current research focuses on investigating the mechanisms by which cells sense and transduce mechanical forces into biochemical signals, and it has emerged that the extent to which an imposed mechanical load can elicit changes in cell behaviour partly depends on the distribution of forces within the structural components of the cell. The mechanisms by which cells resist deformation under imposed mechanical loads, and the contribution of each cellular component to structural stability, are therefore significant questions in cell mechanics.

Mechanical models could give insight into the structural properties of adherent cells. Previous modelling approaches involve either attributing the primary structural role to the cytoskeleton, or treating the cytoplasm as a continuum. The first category of models includes the tensegrity approach, which views the cytoskeleton as a network of microfilaments and microtubules that distribute forces within the cell through a balance of compression and tension (Ingber, 1997; Stamenovic et al., 1996). By attributing a central role to cytoskeleton contractile forces, the tensegrity approach has described many aspects of cell deformability including non-linear features of cellular structural behaviour (Wang et al., 2001). In the second category of models, the continuum approach has been used to model blood cells with a cortical membrane and viscous cytoplasm (Evans and Yeung, 1989), airway epithelial cells in a 2D finite element model (Kamm et al. 2000), the deformation of a chondrocyte within its extracellular matrix (Guilak and Mow, 2000), and cell deformation in magnetocytometry with a viscoelastic representation of the membrane/cortex and cytoskeleton (Karcher et al., 2003). The third category includes models of the cytoskeleton as a percolation network of fibres in a viscous cytoplasm, where the fibres recombine via linker molecules to transmit forces across the cytoskeleton (Shafrir and Forgacs, 2002; Head et al., 2003).

Charras and Horton (2001) have suggested a model comprising a cytoskeleton tethered to both membrane and nucleus components to explain their observations of the effects of cytoskeletal disruption. It has also been proposed by Hochmuth (2000) that "the modelling of cellular deformation in the future may combine elements of both continuum models and tensegrity models". The present paper describes the development a model of a eukaryotic cell adherent to a substrate using the finite element method, incorporating those cellular components that are likely to be structurally significant, i.e. nucleus, 
cytoplasm, membrane components, and a cytoskeleton network of filaments and tubules. The aim is to determine whether or not we can successfully describe the non-linear structural behaviour of an adherent cell, including non-linear stiffening, variable compliance along the cell surface, the influence of tension (i.e., prestress) in the actin filament network, alterations in stiffness during spreading, and the contribution of each structural component to the cellular stability/stiffness. If this is possible then we would have a prima facie case for using such models as predictive tools in mechanobiology.

\section{Model Geometry}

Methods

A finite element model was created to represent the shape of a rounded cell adherent to a substrate in various spread configurations. The geometry was based on images of spreading fibroblasts (Fig. 1). The cell immediately post attachment has its most rounded configuration (Fig. 2a) and consists of a cytoskeleton incorporating microfilaments and microtubules, together with cytoplasm, nucleus and membrane components. Similar models were created to represent the adherent cells at increasingly spread shapes (Fig. $2 b-2 f)$. The internal cytoskeleton consists of a tensegrity network of six compression-bearing struts (two in each orthogonal direction) and twenty-four tensional cables representing the aggregate behaviour of microtubules and microfilaments respectively. The end of each strut connects with four cables, creating 12 common nodes representing 'receptor' sites where actin filaments cluster at adhesion complexes in adherent cells. In the increasingly spread models (Fig. 2a-2f), the microtubule and microfilament elements are altered in length and position as these nodes are re-positioned downwards to comply with the spread shapes. New surface locations for the 'receptor' nodes are established by firstly determining the nodal positions that maintain the original vertical proportions of the internal cytoskeleton structure in the rounded configuration shown in Fig. 2a, i.e. three horizontal planes formed by nodes 4, 5, 6 nearest the substrate, nodes 7, 8, 9 next, and nodes 10, 11, 12 farthest from the substrate (Fig. 2a shows the original positions of these nodes). Radial planes defined by the central axis and the original nodal positions are then established; the intersection of these planes with each new model surface yields surface lines along which the new nodal positions must lie. Points of intersection of these surface lines with each of the three horizontal planes established in each model yield nine new nodal positions of the cytoskeleton nodes when repositioned to conform to the spread shape of each model. The nodes of the membrane are coincident with nodes of the underlying cytoplasm and with nodes of the cytoskeleton at receptor sites. Contact
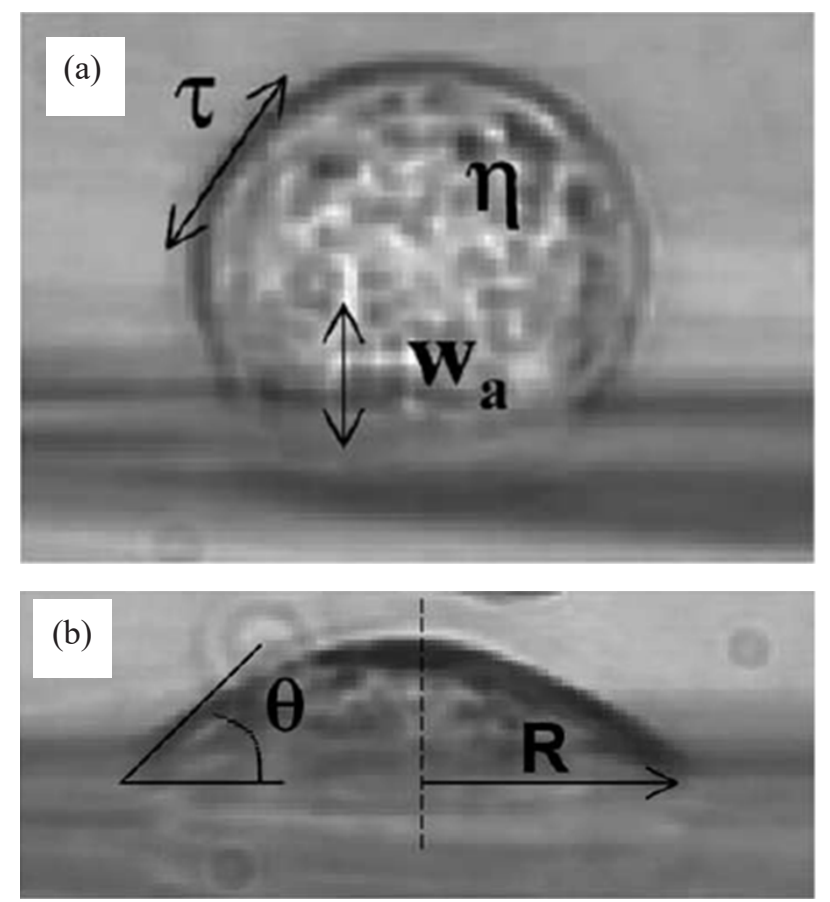

Figure 1. Images of a spreading chick embryo fibroblast adherent on a glass microplate for (a) 5 minutes, and (b) after 3 hours, adapted with permission from Frisch and Thoumine (1999). The dimensions of the finite element models developed (Table 1) correspond to contact angles $(\theta)$ and contact radii (R) highlighted in (b).

radii and heights of each of the finite element models are provided in Table 1. The corresponding cell volumes are $\sim 3,000 \mu \mathrm{m}^{3}$ and the nucleus in each model formed from an ellipse with a major axis of $8 \mu \mathrm{m}$, minor axis of $5 \mu \mathrm{m}$, and a distance of $2 \mu \mathrm{m}$ from the substrate, also based on experimental observations by Thoumine et al. (1999).

The model is developed using ANSYS (Palo Alto, CA, USA). The cytoplasm and nucleus elements are meshed with 4-node lower-order tetrahedral elements. The membrane is meshed with 3-node shell elements. Microtubule struts and microfilament cables are meshed with single link elements (a three-dimensional spar element with bilinear stiffness matrix), compression-only and tension-only respectively.

\section{Constitutive modelling of cellular components}

Material properties for each of the cell components are not known precisely and can only be estimated from various sources (see Table 2). The cytoplasm and nucleus are here treated as linear elastic and isotropic continua. The elastic modulus of the cytoplasm is chosen as $100 \mathrm{~Pa}$ (Kamm et al., 2000), while the nucleus is chosen as $400 \mathrm{~Pa}$,

\begin{tabular}{|l|c|c|c|c|c|}
\hline & Cytoplasm & Nucleus & Membrane $^{\S}$ & Microtubules $^{\S}$ & Microfilaments $^{\S}$ \\
\hline Elastic modulus (Pa) & $100^{*}$ & $400 \dagger$ & $10^{3}$ & $1.2 \times 10^{9}$ & $2.6 \times 10^{9}$ \\
\hline Poisson's ratio (v) & $0.37 \ddagger$ & $0.37 \ddagger$ & 0.3 & 0.3 & 0.3 \\
\hline
\end{tabular}

†Shin and Athanasiou, 2001, †Guilak et al., 2000, " Kamm et al., 2000, ${ }^{\S}$ Gittes et al., 1993.

Table 2. Material properties assigned to the cellular components in each model. 
(a)
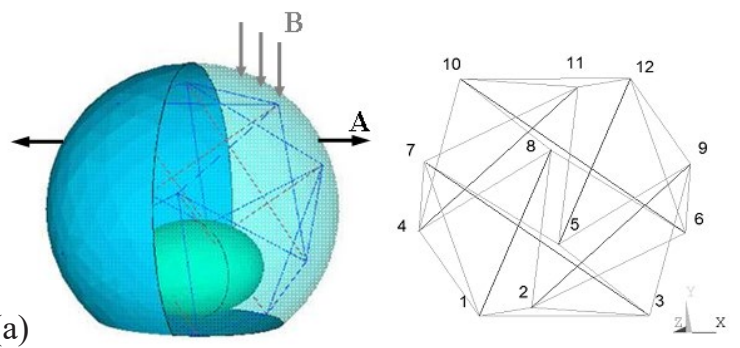

(c)

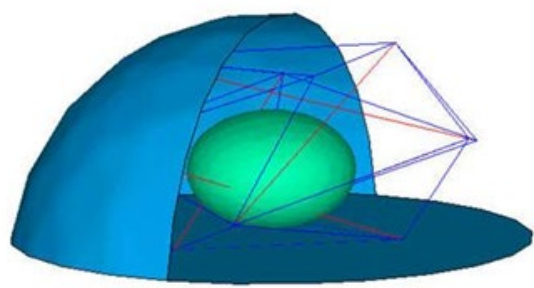

(e)

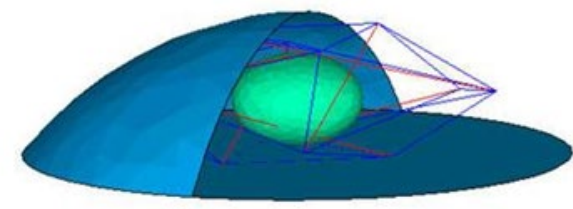

(b)

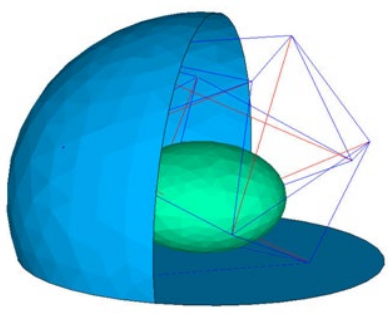

(d)

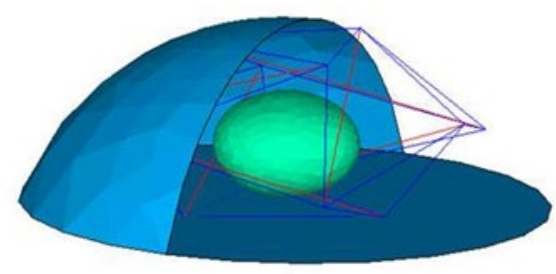

(f)

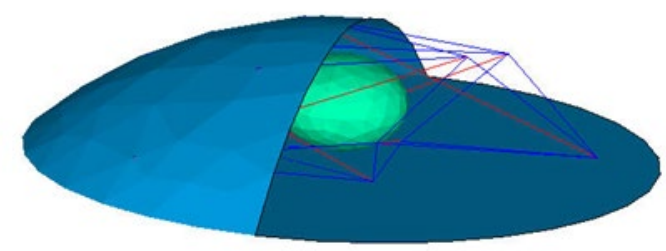

Figure 2. Three-dimensional finite element models (a) to (f) of adherent cells at increasingly spread shapes to the final configuration in Fig 1b. Each model comprises membrane, nucleus, cytoplasm, and cytoskeleton components. The cytoplasm is omitted for clarity in all models except model (a). Also shown is the internal cytoskeleton of model (a) consisting of a 3-dimensional tensegrity network of interconnected microtubule (dark lines) and microfilament elements (light lines). Loading conditions applied to the models involve either (A) horizontal forces (50 $\mathrm{pN}$ ) applied to two surface nodes in opposite directions, or (B) the application of vertical indentation forces (50 $\mathrm{pN})$ to membrane surface nodes.

$\sim$ four times stiffer than the cytoplasm, as reported by Guilak et al. (2000). Poisson's ratio value (v) for both nucleus and cytoplasm is chosen as 0.37 (Shin and Athanasiou, 1999). In the model developed by Kamm et al. (2000) epithelial cell membrane elasticity was taken to be $10^{7} \mathrm{~Pa}$ with a thickness of $6 \mathrm{~nm}$. Since in that study, it was concluded that the membrane elastic properties were over-estimated, a lower value of $10^{3} \mathrm{~Pa}$ (with $v=0.3$ ) is selected for the present model, while maintaining the same membrane thickness. Gittes et al. (1993) measured the flexural rigidity (bending stiffness) of microtubules and microfilaments subjected to thermal fluctuations. The properties applied to the cytoskeleton components, i.e. the microtubules and microfilaments are based on the elasticity values calculated from these bending stiffness values under the assumption of isotropy and homogeneity; the microtubules are assigned corresponding cross-sectional areas of $190 \mathrm{~nm}^{2}$, while the microfilaments are thinner with an area of $18 \mathrm{~nm}^{2}$.

\section{Initial constraints}

In all models with the various degrees of spreading (Fig. $2 \mathrm{a}$ to $2 \mathrm{f}$ ), nodes at the cell-substrate interface, including the three receptor nodes that establish a cell-substrate plane in each model (nodes 1, 2, 3 in Fig. 2a), are constrained in all three translational degrees of freedom. Therefore the constrained points are analogous to focal adhesion sites in adherent cells. Initial constraints in each analysis also involve application of a prestress (initial strain) to the microfilament

\begin{tabular}{|c|c|c|}
\hline Model & Contact radius $(\mu \mathrm{m})$ & Cell Height $(\mu \mathrm{m})$ \\
\hline $\mathbf{a}$ & 6 & 14 \\
\hline b & 8.5 & 12.8 \\
\hline $\mathbf{c}$ & 11.2 & 10.6 \\
\hline $\mathbf{d}$ & 14.3 & 10.1 \\
\hline $\mathbf{e}$ & 17.5 & 8.5 \\
\hline $\mathbf{f}$ & 19.2 & 7.6 \\
\hline
\end{tabular}

Table 1. Dimensions of each finite element model (a) to (f) illustrated in Fig. 2.

elements in each of the six models. Prestress is an initial tension that effectively accounts for the contractile effect of acto-myosin sliding mechanisms in the membrane/ cortex region.

Prestress and strain hardening. Prestress values assigned to microfilament elements of model (a) are varied from 0 to $10 \%$ in the first loadstep of each analysis, while subsequent loadsteps involve application of a $100 \mathrm{pN}$ force $(50 \mathrm{pN}$ applied to two membrane surface nodes as illustrated in Fig. 2a, load vectors denoted by ' $A$ '). The displacement of the nodes in each direction due to the applied force yields stiffness $(\mathrm{nN} / \mu \mathrm{m})$ estimates. To investigate strain hardening, the force applied in the second loadstep is increased from 25 to $150 \mathrm{pN}$ while maintaining constant prestress values in the first loadstep.

Parametric variation of material properties. The influence of material properties on stiffness is investigat- 


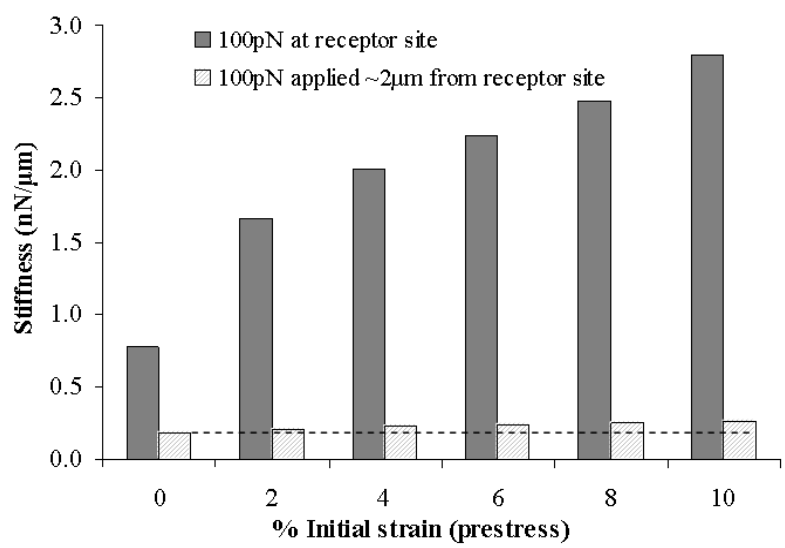

Figure 3. The effect of prestress on cell stiffness values. The dotted line (stiffness at $0 \%$ prestress) is included to illustrate the small increases in stiffness that occur when the force is applied $\sim 2 \mu \mathrm{m}$ from the receptor site.

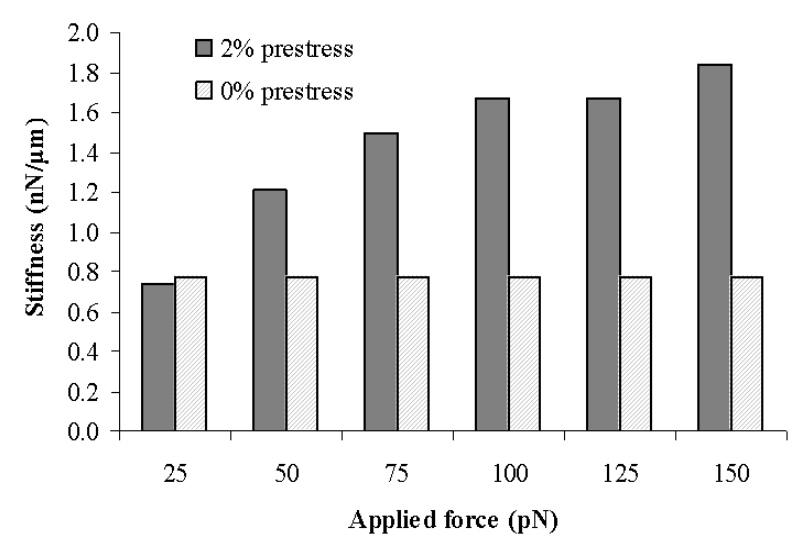

Figure 4. Stiffness versus applied forces. At $0 \%$ prestress in the cytoskeleton there is no strain hardening effect.

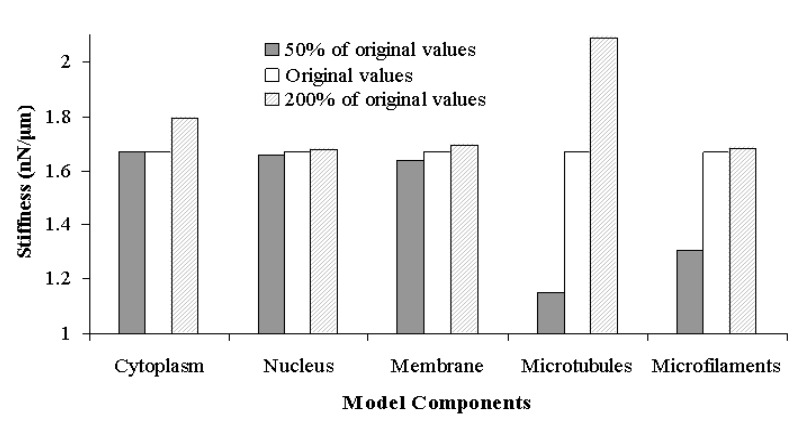

Figure 5. The effect on cell stiffness of altering the Young's modulus of each component from the original values outlined in Table 2.

ed in the unspread cell configuration [Fig. 2a] by modulating the elasticity of each model component by $50 \%$ and $200 \%$ of the original values, and computing displacements in response to $100 \mathrm{pN}$. Poisson's ratio value for the cytoplasm is also altered from 0.37 to 0.25 and 0.49 in order to determine the effect, if any, of the degree of cytoplasm incompressibility. Constant prestress values are maintained during the parameter study.



Figure 6. Variable stiffness on application of vertical indentations of $50 \mathrm{pN}$ along the surface of all models but for model (d) (does not support $50 \mathrm{pN}$ at a distance from the receptor site).

Variable compliance of the cell. All six degrees of spread cell configuration [Fig. 2a to $2 \mathrm{f}$ ] are used to investigate differences in rigidity along the model membrane surface. Again prestress is maintained at a constant value $(2 \%)$ in each model. In each model vertical forces $(50 \mathrm{pN})$ are applied to a receptor node, and at various distances $(<5$ $\mu \mathrm{m}$ ) from the receptor node (as shown in Fig. 2, load vectors denoted by ' $\mathrm{B}$ '). This essentially mimics indentation as applied by atomic force microscopy.

Spreading effects. All six models are also used to investigate alterations in stiffness as contact radius increases. A force of $100 \mathrm{pN}$ is applied as in Fig. 2 - vectors ' $A$ ', but in both $\mathrm{x}$ and $\mathrm{z}$ horizontal directions. Averages of displacements in each direction yield stiffness estimates for each model. Prestress values applied to microfilaments are kept constant in each model, which is in accordance with observations of constant cortical membrane tension in spreading cells (Thoumine et al., 1999).

\section{Results}

When forces in the $\mathrm{pN}$ range are applied to the cell, the model computes small displacements on the order of nanometers. The stiffness values obtained are in a similar range (from $0.5-3 \mathrm{nN} / \mu \mathrm{m}$ ) whether the model is resisting horizontal forces or vertical indentations. As prestress is increased linearly, a non-linear increase in stiffness arises (Fig. 3). This effect of prestress on cellular stiffness is also seen when the force is applied at a distance from the receptor site.

It is also evident that, as the applied force is increased, the stiffness of the cell increases, and this hardening non-linearity disappears in the absence of a prestress (Fig. 4). Since the amount of compression borne by microtubules has been the subject of debate (Ingber et al., 2000; Heidemann et al., 1999) the microtubule components were deactivated in the model illustrated in Fig. 2(a), effectively modelling the treatment of cells with microtubule inhibitors such as colchicine. It was found that, at $1 \%$ prestress, 

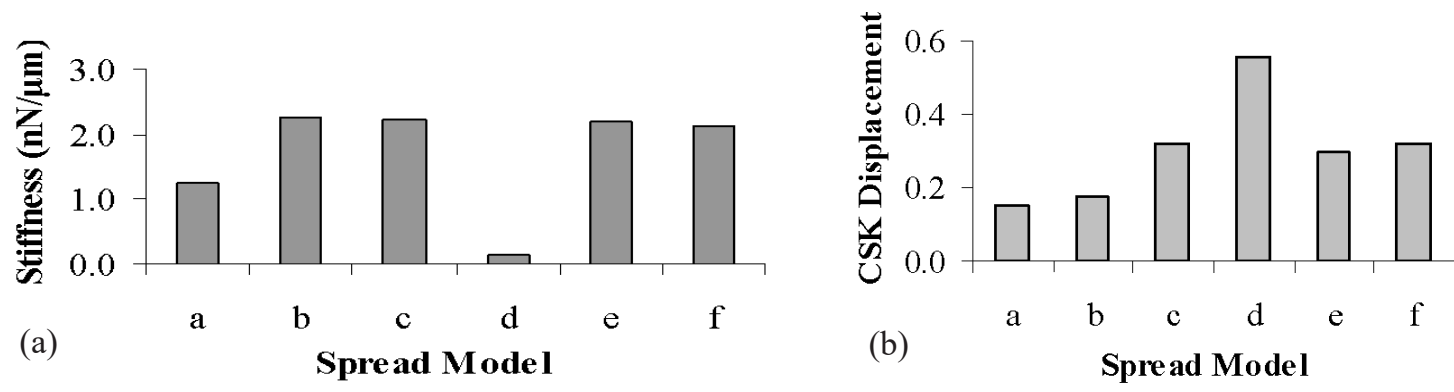

Figure 7. (a) Alterations in stiffness as contact radius increases from models (a) to (f). Graph (b) shows the corresponding cytoskeleton $(\mathrm{CSK})$ displacements $(\mu \mathrm{m})$ due to prestress prior to application of horizontal forces that yield stiffness values.

the decrease in stiffness was $\sim 77 \%$. This finding corresponds to experimental observations of significant losses of structural stiffness upon disruption of microtubules (Stamenovic et al., 2002) and of microtubule buckling in response to increased cell contraction (Wang et al., 2001). Incompressibility of the cytoplasm (i.e. Poisson's ratio, $v$ ) has a significant effect in the model; increasing to 0.49 increases stiffness by $45 \%$, whereas decreasing to 0.25 decreases stiffness by only $\sim 4 \%$.

The cell stiffness changed greatly with changes in the stiffness of certain cell components. Microtubule components have the largest influence on structural stiffness, followed by microfilaments and cytoplasm properties (Fig. 5). The properties of the membrane and nucleus components have a small influence on structural stiffness relative to other components.

In each increasingly spread model applying vertical forces yields differences in compliance along the model surface, i.e. compliance increases with distance from receptor sites (Fig. 6).

As contact radius increases in models (a) to (f) stiffness increases initially, followed by a drop in stiffness in model (d) and a final increase in model (f), as shown in Fig. 7a. Therefore the stiffness is not predicted to change systematically with cell spreading. The corresponding cytoskeleton displacements due to prestress in the first loadstep in each model (Fig. 7b) illustrate slight increases in displacement to a maximum in model (d), which may be related to the trend in stiffness values in Fig. 7a.

\section{Discussion}

The non-linear increases in cell stiffness as a result of increasing prestress exhibited by the model is attributed to the internal cytoskeleton - the effect is also seen when forces are applied at a distance from the underlying cytoskeleton, although the effect is less pronounced. Experimental observations of such an effect in adherent cells have been reported by Pourati et al. (1998) and Wang et al., (2002). The strain hardening effect captured by the model is also due to the cytoskeleton and corresponds to experimental observations of increases in cell stiffness in response to increasing loads applied using micropipette techniques (Sato et al., 1990) and a magnetic twisting device (Wang et al., 2001). Therefore the results of our finite element model concur qualitatively with the experimental findings that the internal cytoskeleton plays a key role in determining the structural properties of the model.

With the exception of the cytoskeleton, the cytoplasm has the largest influence on the overall resistance to deformation even though its elasticity is significantly less than that of the nucleus and membrane (Fig. 5). This, of course, may be attributed to the large volume occupied by the cytoplasm relative to other components. This indicates the importance of determining correctly the incompressibility of the cytoplasm, particularly since a wide range of values have been reported from experimental studies: Shin and Athanasiou (1999) have reported a value of 0.37 for osteoblasts using a cytoindentation method and linear biphasic finite element analysis; Mathur et al. (2001) and Kamm et al. (2000) use values of up to 0.49 to describe cytoplasm incompressibility. The membrane, despite its small thickness has a larger influence on stiffness than does the nucleus. Increases in the elasticity of the microfilament components have a small effect, while a decrease is more notable since this compromises the greater structural contribution of the microtubules. This influence of microfilament properties on structural stiffness further confirms the view that the contractile force transmitted by actin filaments in adherent cells has a large effect on cellular structural behaviour. Other recent publications have also suggested the importance of prestress in giving the cell the structural stability to resist cellular deformation (Wang et al., 2002). Changes in microtubule properties have the largest influence on the structural stiffness of the cell - this is attributed to their larger cross-sectional area, their positioning, and their role as compression bearing elements in the model.

It has been observed that adherent cells increase their resistance to deformation (or stiffness) as they become more spread on a substrate (Thoumine et al., 1999). The models developed here do not conclusively reflect these findings (Fig. 7). The lack of such stiffening in our model may reflect the simplistic nature of how we have modelled the re-arrangement of the cytoskeleton as the cell spreads. In this respect, the percolation network models mentioned in the Introduction (Shafrir and Forgacs, 2002; Head et al., 2003) may prove superior. However it is evident that there may be a relation between stiffness and the cytoskeleton in each model, i.e. it may be said that the movement of the cytoskeleton in response to arbitrary prestress value is related to the final stiffness value (Fig. 7), e.g. high CSK displacement results in low stiffness (as in model (d)) and 
vice versa (model (b)). Such results emphasise again the influence of the cytoskeleton on overall structural behaviour. Interestingly, to explain their observations, it has been suggested by Thoumine et al. (1999) that stiffening upon spreading may be correlated with structural re-organisation of the cytoskeleton during the spreading process; this further indicates the importance of modelling cytoskeletal re-arrangements in future work.

We also report that compliance varies considerably along the surface of each model, with the highest resistance to indentation at receptor sites due to the underlying cytoskeleton. The stiffness values decrease considerably at short distances from receptor sites and converge to a range of lower stiffness values in each spread model (Fig. 6). This corresponds to variations in elasticity values determined when indenting into the surface of adherent cells using atomic force microscopy; e.g. local elasticity values vary from $\sim 0.5 \mathrm{kPa}$ to $170 \mathrm{kPa}$ if indentation is applied in the region of stress fibres or actin filaments (Mathur et al. 2001; Domke et al. 1996). Quantitative confirmation of the present model has been addressed in McGarry and Prendergast (2003). In that work, we applied displacement boundary conditions that reflect the geometry of an indenting AFM probe and, together with the use of an appropriate theoretical relation (Hertz, 1881; Sneddon, 1965), we were able to determine elasticity values in the range of those reported from experiments.

By accounting for the role of all structurally relevant components in maintaining a cell's stability, while incorporating non-linear aspects of adherent cell behaviour that are due to an interconnected cytoskeleton, the models developed in this paper can be viewed as a fusion of two contrasting previous approaches, the stress-supported tensegrity approach (Ingber, 1997) and the continuum approach (Kamm et al., 2000). A benefit of the approach to repositioning the tensegrity-based cytoskeleton to increasingly spread shapes is related to the fact that the orientation, positioning and length of internal cytoskeleton components is altered by polymerisation, and by clustering of actin filaments during spreading as adhesion bonds form between intra- and extra-cellular structural proteins such as vinculin, talin, and fibronectin (Alberts et al., 1989). It has also been suggested by Gittes et al. (1993) that to account for the seemingly excessively rigid mechanical properties measured, sliding and movement may occur between filaments and tubules. The material properties chosen to represent the cytoskeleton have been used in previous models (Stamenovic and Coughlin, 2000), while similar values for flexural rigidity have been estimated theoretically by Ben-Avraham and Tirion (1995), and are in agreement with other experimental work (Tsuda et al., 1996). The role of intermediate filaments was not included since their contribution to cellular rigidity only becomes significant above 20\% strain (Janmey et al., 1991; Wang and Stamenovic, 2000).

We set out to develop a computational model to provide quantitative predictions of the non-linear structural behaviours that are attributed to the cytoskeleton components, and the relative importance of various cellular components in resisting imposed mechanical loads. The idealised geometry and linear elastic properties used are sufficient to explore the static response to imposed loads, while the need for morphologically accurate model geometries, with time-dependent material properties can be addressed. By application of appropriate loading conditions, the model could also be used to compute cell deformation due to various mechanical stimuli in vitro (such as fluid flow, substrate strain, microgravity) in order to understand the biomechanical origins of differences in observed cell response. It could also be used as a means of comparing the effect of various single-cell stimulation methods (such as optical tweezers, atomic force microscopy, or microplate manipulation).

\section{Acknowledgements}

Funding provided by the Programme for Research in Third Level Institutions (PRTLI), administered by the Higher Education Authority, and by the BITES (Biomechanical Interactions in Tissue Engineering and Surgical Repair) project funded under the $5^{\text {th }}$ Framework programme of the European Commission.

\section{References}

Alberts B, Bray D, Lewis J, Raff M, Roberts K, Watson JD (1989) Molecular Biology of the Cell. Garland Publishing, New York and London, 2nd Edition.

Ben-Avraham D, Tirion MM (1995) Dynamic and elastic properties of F-actin: a normal modes analysis. Biophys J 68: 1231-1245.

Carter DR, Beaupré GS (2003) Skeletal Function and Form. Cambridge University Press.

Charras GT, Horton MA (2002) Single cell mechanotransduction and its modulation analyzed by atomic force microscopy indentation. Biophys J 82: 2970-2981.

Chen CS, Mrksich M, Huang S, Whitesides GM, Ingber DE (1997) Geometric control of cell life and death. Science 276: $1425-1428$.

Chicurel ME, Chen CS, Ingber DE (1998) Cellular control lies in the balance of forces. Curr Biol 10: 232-239.

Domke J, Dannohl S, Parak WJ, Müller O, Aicher WK, Radmacher M (2000) Substrate dependent differences in morphology and elasticity of living osteoblasts investigated by atomic force microscopy. Coll Surf B: Biointerfaces 19: 367-379.

Evans E, Yeung A (1989) Apparent viscosity and cortical tension of blood granulocytes determined by micropipette aspiration. Biophys J 56: 151-160.

Frisch T, Thoumine O (2002) Predicting the kinetics of cell spreading. J Biomech 35: 1137-1141.

Gittes F, Mickey B, Nettleton J, Howard J (1993) Flexural rigidity of microtubules and actin filaments measured from thermal fluctuations in shape. J Cell Biol 120: 923934.

Guilak F, Mow C (2000) The mechanical environment of the chondrocyte: a biphasic finite element model of cell-matrix interactions in articular cartilage. J Biomech 33: 1663-1673.

Guilak F, Tedrow JR, Burgkart R (2000) Viscoelastic properties of the cell nucleus. Biochem Biophys Res Commun 269: 781-786. 
Head DA, Levine AJ, MacKintosh FC (2003) Deformation of cross-linked semi-flexible polymer networks. Phys Rev Lett 91: 108102-1.

Heidemann SR, Kaech S, Buxbaum RE, Matus A (1999) Direct observations of the mechanical behaviours of the cytoskeleton in living fibroblasts. J Cell Biol 145: 109-122.

Hertz H (1881) Über den Kontakt elastischer Körper. Journal für die reine und angewandte Mathematik 92: 156.

Hochmuth RM (2000) Micropipette aspiration of living cells. J Biomech 33: 15-22.

Ingber DE (1997) Tensegrity: the architectural basis of cellular mechanotransduction. Annu Rev Physiol 59: 575-599.

Ingber DE, Heidemann SR, Lamoroux P, Buxbaum RE (2000) Opposing views on tensegrity as a framework for understanding cell mechanics. J Appl Physiol 89: 1663 1670 .

Janmey PA (1998), The cytoskeleton and cell signaling: component localization and mechanical coupling. Physiol Rev 78: 763-781

Janmey PA, Eutenauer U, Traub P, Schliwa M (1991) Viscoelastic properties of vimentin compared with other filamentous biopolymer networks. J Cell Biol 113: 155160 .

Kamm RD, McVittie AK, Bathe M (2000) On the Role of Continuum Models in Mechanobiology. ASME International Congress - Mechanics in Biology 242: 1-9.

Karcher H, Lammerding J, Huang H, Lee RT, Kamm RD, Kaazempur MR (2003) A three-dimensional viscoelastic model for cell deformation with experimental verification. Biophys J 85: 3336-3349.

Mathur AB, Collinsworth AM, Reichert WM, Kraus WE, Truskey GA (2001) Endothelial, cardiac muscle and skeletal muscle exhibit different viscous and elastic properties as determined by atomic force microscopy. J Biomech 34: 1545-1553.

McGarry JG, Prendergast PJ (2003) A finite element model of an adherent cell: comparison of simulated cell indentation with atomic force microscopy experiments. Proceedings of the International Congress on Computational Bioengineering (Eds.: M. Doblaré, M. Cerrolaza, and H. Rodrigues), pp. 552-559, University of Zaragoza, Spain, 2003

Pourati J, Maniotis A, Spiegel D, Schaffer JL, Butler JP, Fredberg JJ, Ingber DE, Stamenovice D, Wang N (1998) Is cytoskeletal tension a major determinant of cell deformability in adherent endothelial cells? Am J Physiol Cell Physiol 274: C1283-1289.

Sato M, Theret DP, Wheelerm LT, Ohshima N, Nerem RM (1990) Application of the micropipette technique to the measurement of cultured porcine aortic endothelial cell viscoelastic properties. J Biomech Eng 112: 263-268.

Shafrir YG, Forgacs G (2002) Mechanotransduction through the cytoskeleton. Am J Physiol Cell Physiol 282: C479-486.

Shin D, Athanasiou K (1999) Cytoindentation for obtaining cell biomechanical properties. J Orthop Res 17: 880-890.
Sneddon IN (1965) The relation between load and penetration in the axisymmetric Boussinesq problem for a punch of arbitrary profile. Int J Eng Sci 3: 47-57.

Stamenovic D, Coughlin MF (2000) A Quantitative Model of Cellular Elasticity Based on Tensegrity. J Biomech Eng, ASME Transactions 122: 39-43.

Stamenovic D, Fredberg JJ, Wang N, Butler J, Ingber DE (1996) A microstructural approach to cytoskeletal mechanics based on tensegrity J Theor Biol 181: 125-136.

Stamenovice D, Mijailovich SM, Tolic-Norrelykke IM, Chen J, Wang N (2002) Cell prestress. II. Contribution of microtubules. Am J Physiol Cell Physiol 282: C617-624.

Thoumine O, Cardoso O, Meister J-J (1999) Changes in the mechanical properties of fibroblasts during spreading: a micromanipulation study. Eur Biophys J 28: 222-234.

Tsuda Y, Yasutake H, Ishijima A, Yanagida T (1996) Torsional rigidity of single actin filaments and actin bond breaking force under torsion measured directly by in vitro micromanipulation. Proc Natl Acad Sci USA 93: $12937-$ 12942.

Wang N, Stamenovic D (2000) Contribution of intermediate filaments to cell stiffness, stiffening and growth. Am J Physiol Cell Physiol 279: C199-194.

Wang N, Naruse K, Stamenovic D (2001) Mechanical behaviour in living cells consistent with the tensegrity model. Proc Natl Acad Sci USA 98: 7765-7770.

Wang N, Tolic-Norrelykke IM, Chen J, Mijailovich SM, Butler JP, Fredberg JJ, Stamenovice D (2002) Cell prestress I. Stiffness and prestress are closely associated in adherent contractile cells. Am J Physiol Cell Physiol 282: C606-616.

\section{Discussion with Reviewers}

B. Ashton: When a cell attaches to a substrate integrins in the membrane become linked externally to their ligand on the substrate and to the cytoskeleton internally. Is it legitimate to continue to model the biomechanical properties of this complex as the sum of the individual components - membrane, microfilaments?

Authors: The modelling approach we have taken is legitimate in so far as it approximates the distribution of integrin-ligand links as a continuum. However, the number and strength of integrin-ligand links influences the clustering of actin filaments at focal adhesion complexes which in turn effects the distribution, length, and orientation of the cytoskeletal elements which, we assume, dictate cell biomechanical properties. Therefore, modelling the "individual components" as a continuum is a simplification which would have considerable influence on modelling the dynamic process of cell spreading rather more so than on determining the biomechanical properties of an adherent cell.

Essentially what we have done in our model might be considered as taking a "snapshot" of the cytoskeletal network in increasingly spread configurations without incorporating the mechanisms (such as integrin-ligand links and associated biophysical pathways) that drive these changes. In summary, by manually repositioning the 
cytoskeleton our approach does not model the dynamics of cell spreading but rather the consequences of it in terms of the cellular biomechanical properties.

J. Klein-Nulend: How do the forces applied in your model compare quantitatively to the shear stress applied to cells in fluid flow experiments, or to the forces applied in experiments in which cell response has been manipulated with optical tweezers or atomic force microscopy techniques? Authors: From literature it seems that the localised forces required to elicit biological responses in single adherent cells are higher in magnitude (on the order of $\mathrm{nN}$ 's) than the forces we have applied to our model (on the order of $\mathrm{pN}$ 's). For example, pulling magnetically on microbeads adherent to cell receptors with a force of $4.4 \mathrm{pN}$ does not alter calcium response (Wu et al., 1999), whereas higher forces of $10 \mathrm{nN}$ applied by atomic force microscopy indentations can modulate intracellular calcium response (Charras and Horton, 2002). However, we have computed that displacements of our model due to physiological levels of shear stress known to cause alterations in cellular function (e.g. Mullender et al., 2004) are of the same magnitude as those displacements caused by the forces applied to our model here (on the order of nm's). This difference in magnitude suggests that adherent cells may distinguish between and respond differently to whole-cell (e.g. fluid flow) and localised methods of cell stimulation (e.g. AFM, optical tweezers).

J. Klein-Nulend: In your model, material properties are estimated from various sources for different cell types. Can you comment on this variation in properties of different cell types and how such variation would impact on the structural behaviour of the model?

Authors: Differences in material properties have been reported for various cell types - Mathur et al. (2001) have reported elastic moduli of $1.4 \mathrm{kPa}$ for endothelial cells, $24.7 \mathrm{kPa}$ for skeletal muscle cells and $100 \mathrm{kPa}$ for cardiac muscle cells. The properties of our model components can be altered accordingly to describe the structural behaviour of these different cell types, and provided that the relative differences in material properties assigned to each component remain consistent, the structural behaviours reported here will remain valid. Perhaps what is more important is to determine why there is such variation in mechanical properties between cell types? Our findings suggest not only that many aspects of cellular structural behaviour seen in our model depend on the cytoskeleton, but also indicate that such large variations in properties between cell types (as in Mathur et al. (2001) for example) can only (and most likely) arise from differences in the distribution, lengths and orientations of the more rigid cytoskeleton components. This again emphasises the importance of understanding the mechanisms of adhesion bond formation that control the distribution, positioning and polymerisation of cytoskeletal components, and of developing experimental techniques to determine cytoskeletal properties.

J. Klein-Nulend: Could the authors elaborate on why intermediate filaments were not included in the model?

Authors: Our approach to modelling the cytoskeleton focuses on a specific role for microfilaments in transmitting a tension or prestress which is resisted by interconnected microtubules that are in compression. Although intermediate filaments are thought to contribute largely to the mechanical support of the cell (Alberts et al., 1989), particularly at higher strains (Janmey et al., 1991; Wang and Stamenovic, 2000), we are not aware of a specific tensile or compressive role for intermediate filaments or whether they form an interconnected network for force transmission with the other cytoskeletal components. For this reason, we did not explicitly model intermediate filaments, and their contribution to cellular stiffness was simply assumed to be incorporated in the material properties of the cytoplasm.

\section{Additional References}

Mullender MG, El Haj AJ, Yang AJ, van Duin MA, Burger EH, Klein-Nulend J (2004) Mechanotransduction of bone cells in vitro - mechanobiology of bone tissue. Med Biol Eng Comp 42: 14-21.

Wu Z, Wong K, Glogauer M, Ellen RP, McCulloch CA (1999) Regulation of stretch activated intracellular calcium transients by actin filaments. Biochem Biophys Res Commun 186: 1212-1219. 